\title{
Pericarditis Constrictiva Tuberculosa. Reporte de un Caso y Revisión de la Literatura
}

\author{
Tuberculous Constrictive Pericarditis. Case Report and Literature Review \\ Oscar Tapia E.
}

TAPIA, E. O. Pericarditis constrictiva tuberculosa. Reporte de un caso y revisión de la literatura. Int. J. Morphol., 30(2):696-700, 2012.

RESUMEN: La pericarditis tuberculosa (PT) representa una rara manifestación extrapulmonar de tuberculosis (TBC), que se encuentra en aproximadamente el 1\% de las autopsias por TBC y 1-2\% de casos de TBC pulmonar, estando su presentación estrechamente asociado con la infección por VIH. El compromiso pericárdico ocurre habitualmente por diseminación linfática retrógrada o diseminación hematógena desde un foco pulmonar primario, pudiendo clínicamente manifestarse como derrame pericárdico, pericarditis constrictiva o un patrón mixto. El presente trabajo, describe los hallazgos clínicos y morfológicos de un caso de PT en un sujeto inmunocompetente de 78 años, con diagnóstico de PT constrictiva efectuado en el Hospital Hernán Henríquez Aravena de Temuco.

PALABRAS CLAVE: Pericarditis; Tuberculosis; Tuberculous pericarditis.

\section{INTRODUCCIÓN}

La pericarditis tuberculosa (PT) es una manifestación extrapulmonar rara, que se encuentra en aproximadamente el $1 \%$ de las autopsias por tuberculosis (TBC) y $1-$ $2 \%$ de casos de TBC pulmonar (Mayosi et al., 2005; Fowler, 1991). En algunos países de África y Asia la PT es la causa más frecuente de pericarditis, llegando a representar casi el $70 \%$ de los casos en algunas áreas geográficas de África, mientras que en países desarrollados esta representa tan solo el 4\% de los casos de pericarditis; diferencias epidemioló-gicas atribuibles a la infección por el VIH, reportándose que hasta el $81 \%$ de los sujetos con PT son VIH (+) (Mayosi et al., 2003; Fowler; Reuters et al., 2005; Mayosi et al., 2005).

El compromiso pericárdico ocurre habitualmente por diseminación linfática retrógrada, desde los linfonodos paratraqueales, peribronquiales y mediastínicos, o por diseminación hematógena desde un foco pulmonar primario, pudiendo clínicamente manifestarse como derrame pericárdico, pericarditis constrictiva o un patrón mixto (Mayosi et al., 2005).

La mortalidad de la PT fluctúa entre $20-40 \%$, ocurriendo el $16 \%$ de estas muertes en la fase aguda como resultado del taponamiento cardíaco (Mayosi et al., 2005).
En el presente trabajo, se describen los hallazgos clínicos y morfológicos de un caso de PT en un sujeto inmunocompetente de 78 años, con diagnóstico de PT constrictiva efectuado en el Hospital Hernán Henríquez Aravena de Temuco, Chile.

\section{CASO CLÍNICO}

Hombre de 78 años, obrero, con antecedentes TBC pulmonar tratada hace aproximadamente 40 años, hipertensión arterial y diabetes mellitus tipo 2 en tratamiento.

Consultó en servicio de urgencia luego de sufrir síncope. El paciente refiere presentar dificultad respiratoria progresiva desde hace 6 meses, llegando a estar actualmente en capacidad funcional III, asociado a edema de miembros inferiores, sudoración nocturna y baja de peso. Al examen físico se constata un sujeto enflaquecido, polipneico, con disnea a esfuerzos mínimos, ingurgitación yugular, signología de derrame pleural bilateral, ascitis leve y edema marcado de miembros inferiores y región lumbar.

Se realizó radiografía de tórax que mostró derrame pleural bilateral y silueta cardiaca aumentada de tamaño. 
Los exámenes de sangre demostraron hemograma normal (hematocrito 39,1\%, VCM: 86 fL y HCM 28 pg) y gasometría arterial con acidosis mixta e hipoxemia ( $\mathrm{pH} 7.31$, PCO2 47,5 mm/hg, PO2 $71 \mathrm{~mm} / \mathrm{hg}$ y HCO3 22,1 mmol/L) Se decidió hospitalizar al paciente con diagnóstico de insuficiencia cardiaca descompensada.

La ecotomografía abdominal evidenció ascitis de leve a moderada cuantía, y hepatoesplenomegalia congestiva. Se efectuó manejo médico farmacológico para insuficiencia cardíaca descompensada que incluyó diuréticos, inhibidores de la enzima convertidora de la angiotensina y bbloqueadores.

Transcurrido 11 días de hospitalización y escasa mejoría clínica se efectuó ecocardiograma transtorácico que mostró derrame y engrosamiento pericárdico $(8 \mathrm{~mm})$, aumento leve de la presión sistólica de la arteria pulmonar (29 $\mathrm{mmHg}$ ), sin disfunción diastólica y fracción de eyección del ventrículo izquierdo de $68 \%$. La tomografía axial computada (TC) de tórax y abdomen confirmó el derrame pleural bilateral y acentuado engrosamiento pericárdico (Fig. 1) con ascitis de moderada cantidad. El test de ELISA para VIH resultó negativo. La punción del líquido pericárdico demostró $78 \%$ mononucleares y $22 \%$ polimorfonucleares, proteínas totales de $8,09 \mathrm{~g} / \mathrm{dL}$, glucosa $187 \mathrm{mg} / \mathrm{dL}$, albúmina 2,7 gr/dL, LDH 76 U/L, ADA 5,7 UI; negativo para células neoplásicas.

El paciente evoluciona sin mejoría clínica con disfunción ventricular y aumento de la cuantía del derrame pleural y ascitis.

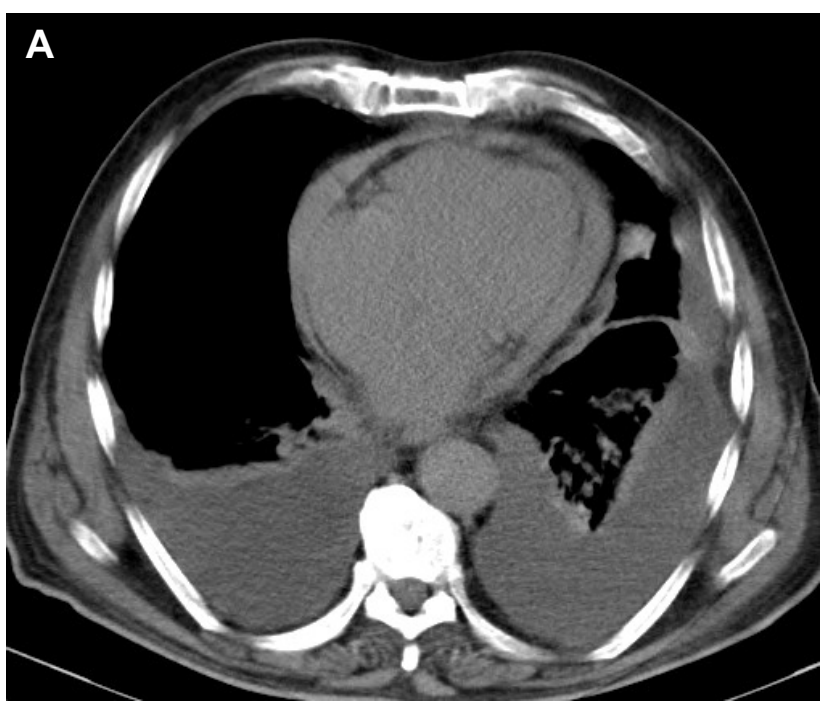

Fig. 1. Tomografía computada (TC) de tórax y abdomen donde se observa derrame pleural bilateral y acentuado engrosamiento pericárdico.
Luego de 18 días se realizó ventana pericárdica obteniéndose tejido para estudio bacteriológico e histopatológico. La biopsia de la pieza quirúrgica mostró pericardio con proceso inflamatorio crónico productivocaseoso, demostrando la tinción de Ziehl-Neelsen presencia de bacilos ácido alcohol resistentes (Fig. 2).

Con este resultado se inicia tratamiento anti-tuberculoso, sin embargo, en los días siguientes el paciente evoluciona con falla renal aguda y mayor compromiso cardiovascular falleciendo finalmente luego de recibir 8 días de terapia anti-TBC.

\section{DISCUSIÓN}

La PT representa una infrecuente forma de manifestación de TBC extrapulmonar, representando en algunos países de África y Asia la causa más frecuente de pericarditis, condición explicada por la alta prevalencia de infección por el VIH, reportándose que entre 50-81\% de los sujetos con PT son VIH (+) (Mayosi et al., 2005; Trautner \& Darouiche, 2001).

El compromiso pericárdico ocurre generalmente por diseminación linfática retrógrada del M. tuberculosis, desde linfonodos peritraqueales, peribronquiales y/o mediastínicos o por diseminación hematógena desde un foco pulmonar primario; siendo infrecuente que la afectación ocurra por vía hematógena, extensión contigua desde un foco pulmonar y/o a partir de una infección secundaria ósea o del tracto genitourinario (Mayosi et al., 2005). En nuestro paciente existía el antecedente de TBC pulmonar antigua tratada, sin embargo, ante la ausencia de signología respiratoria no se descartó una reinfección o reactivación pulmonar, por lo que la posible etiopatogenia pudiese explicarse por la reinfección o reactivación de un foco pulmonar o linfático.

Morfológicamente es posible reconocer 4 estadios evolutivos de la PT: 1. Exudación fibrinosa con polimorfonucleares, abundantes micobacterias y formación de granulomas, 2. Derrame hemático con exudado de predominio linfocitario, 3. Organización y engrosamiento pericárdico y 4. Pericarditis constrictiva; pudiendo variar los hallazgos morfológicos de acuerdo al estado inmunológico del paciente (Mayosi et al., 2005; Reuter et al., 2006); representando el presente caso el estadio final final de la PT.

La PT se puede presentar a cualquier edad, siendo más común entre la tercera y quinta década de la vida, y tres a cuatro veces más frecuente en hombres. Clínicamente exis- 

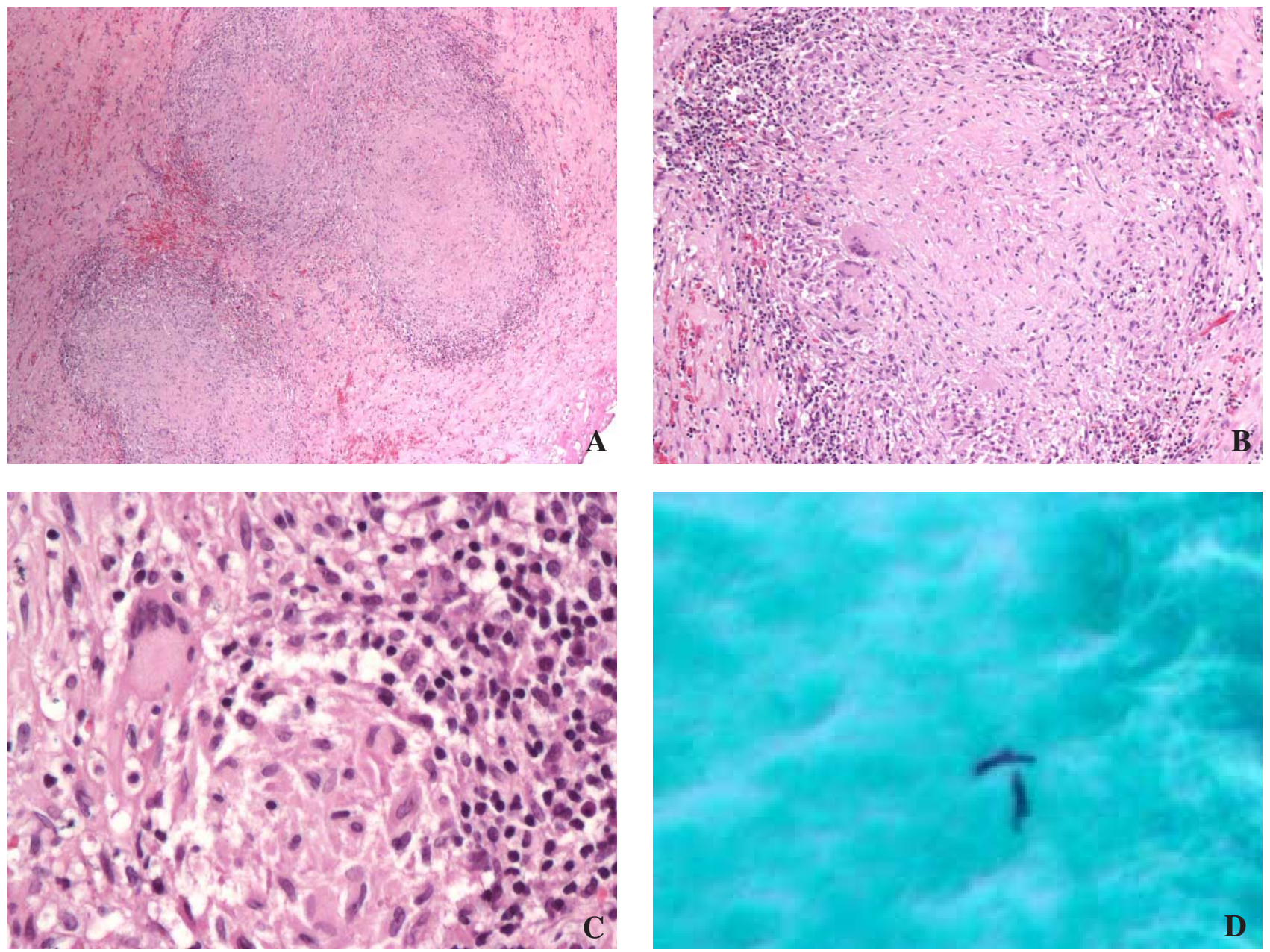

Fig. 2. A (HE, 40x), B (HE, 100x), C (HE, 400x) y D (Ziehl Neelsen, 1000x). A-C: tejido pericárdico con proceso inflamatorio granulomatoso, donde los granulomas presentan un foco central caseoso rodeado de una corona de células epiteloideas dispuesta en forma de empalizada entre las cuales se distinguen células gigantes de Langhans (?) y en la periferia infiltrado inflamatorio linfoplasmocitario. D: presencia de bacilos ácido alcohol resistentes con tinción de Ziehl Neelsen.

ten tres formas de presentación: derrame pericárdico, pericarditis constrictiva y forma mixta. La pericarditis constrictiva por su parte, representa una de las secuelas más graves de la PT, presentándose en el 30-60\% de estos pacientes, en la mayoría de ellos en forma subaguda. Los síntomas de la PT incluyen tos, disnea, dolor torácico, sudoración nocturna, ortopnea, edema y baja de peso. En cuanto a los signos, la cardiomegalia, frote pericárdico, fiebre, taquicardia, pulso paradójico, hepatomegalia, ingurgitación yugular y derrame pleural son reportados; debiendo ser sospechado el compromiso pericárdico en todo paciente con antecedentes o formas activas de TBC (Mayosi et al., 2005; Trautner \& Darouiche).

Los hallazgos en la radiografía de tórax y electrocardiograma son inespecíficos, siendo el ecocardiograma el estudio no invasivo de elección, permitiendo identificar y cuantificar el derrame pericárdico, asociado a un pericardio en- grosado con disminución de los movimientos cardíacos, en ausencia de enfermedad cardíaca valvular e hipertrofia miocárdica (Mayosi et al., 2005; Trautner \& Darouiche). En $80 \%$ de los casos, en la pericardiocentesis se obtiene un exudado hemorrágico con predominio mononuclear, proteínas elevadas y glucosa baja (Mayosi et al., 2005; Mayosi et al., 2003). La enzima adenosin-deaminasa (ADA) es una herramienta útil en el diagnóstico de TBC pericárdica cuando es mayor de 35 UI, reportándose una sensibilidad de $88 \%$, especificidad de $83 \%$, valor predictor positivo de $83 \%$ y valor predictor negativo de 88\% (Lasso \& Pérez, 2009; Tuon et al., 2006). En el caso presentado la radiografía de tórax demostró un aumento de la silueta cardíaca, mientras que la ecocardiografía evidenció un marcado engrosamiento pericárdico, sin embargo, el valor de ADA en el líquido pericárdico resultó dentro de rangos normales; hecho que dificultó aún mas la aproximación diagnóstica. El diagnóstico 
definitivo en tanto se realiza mediante el aislamiento del $\mathrm{M}$. tuberculosis ya sea en un cultivo o examen en fresco de líquido o tejido pericárdico y/o un proceso inflamatorio crónico granulomatoso caseoso en el estudio anatomo-patológico de muestras obtenidas de pericardio, siendo la sensibilidad y especificidad de $87 \%$ y $100 \%$ para la biopsia y $94 \%$ y $100 \%$ para el cultivo; obteniendose un mejor rendimiento en cultivos de tejido (93\% de positividad) que en líquido pericárdico (57\%) (Trautner \& Darouiche; Cegielski et al., 1995); en este paciente los hallazgos histopatológicos fueron compatibles con una etiología tuberculosa, no disponiéndose del resultado del cutivo de tejido antes del fallecimiento. Por otro lado la reacción en cadena de la polimerasa (PCR) para M. tuberculosis resulta de poca utilidad en estos sujetos, dada su pobre sensibilidad en fluidos, fácil contaminación y presencia de falsos positivos (sensibilidad $81 \%$ y especificidad $75 \%$ ) (Mayosi et al., 2005; Trautner \& Darouiche; Cegielski et al.).

El tratamiento de la PT constrictiva se basa en el uso de medicamentos antituberculosos, los que han demostrado disminuir la mortalidad de $80-90 \%$ en la era preantibiótica a cifras que fluctúan entre 8-17\% en sujetos VIH (-) y a 17$34 \%$ en VIH (+). Los esquemas de tratamiento consideran rifampicina, isoniazida, pirazinamida y etambutol durante 2 meses, seguido de isoniacida y rifampicina por 4 meses para así completar un periodo total de tratamiento de 6 meses. Por otro lado, la resolución de la restricción mecánica mediante pericardiectomía debe ser considerada en estos pacientes; existiendo en este punto controversias respecto a que pacientes efectuarla, recomendando algunos autores la pericardiectomía en todos los sujetos una vez iniciado el tratamiento anti-tuberculoso, mientras que otros prefieren reservarla para pacientes que no responden al tratamiento farmacológico, basado estos en estudios que demuestran que la constricción se resuelve en la mayoría de los pacientes luego del tratamiento médico y sólo $25 \%$ de los enfermos requieren pericardiectomía por persistencia o empeoramiento de la constricción luego de 2 años; siendo la mortalidad después de efectuada la pericardiectomía de 3-16\% (Mayosi et al., 2005; Trautner \& Darouiche; Holman \& Willet, 1951; Carson et al., 1974; Sonneberg \& Parker, 1986; Mayosi et al., 2002; Yang et al., 2005). El uso asociado de corticoesteroides por su parte ha demostrado disminuir la mortalidad de la PT y su evolución a la forma constrictiva, del mismo modo la realización de un drenaje pericárdico abierto (ventana pericárdica) logra una menor progresión a pericarditis constrictiva comparado con aquellos enfermos en los que se efectúa pericardiocentesis (4\% vs 9\%) (Yang et al.; Strang et al., 1988; Strang et al., 1987; Dooley et al., 1997).

En resumen, podemos concluir que la pericarditis tuberculosa es una entidad clínico-patológica poco frecuente, que debe ser sospechada en todo paciente con derrame pericárdico, mas aún si se asocia a estados de inmunodepresión; donde el oportuno diagnóstico y precoz inicio del tratamiento farmacológico permitirían la mejoría clínica.

TAPIA, E. O. Tuberculous constrictive pericarditis. Case report and literature review. Int. J. Morphol., 30(2):696-700, 2012.

SUMMARY: Tuberculous pericarditis (TP) is a rare manifestation of extrapulmonary tuberculosis (TBC), found in approximately $1 \%$ of autopsies by TB and $1-2 \%$ of cases of pulmonary TB, while his presentation closely associated with the HIV infection. The pericardial involvement usually occurs by retrograde lymphatic spread or hematogenous spread from a primary pulmonary focus, which may clinically manifest as pericardial effusion, constrictive pericarditis or a mixed pattern. This paper describes the clinical and morphological findings of a TP case in an 78-year old immunocompetent patient, with constrictive TP diagnosed made in the Hospital Hernán Henríquez Aravena in Temuco.

KEY WORDS: Pericarditis; Tuberculosis; Tuberculous pericarditis.

\section{REFERENCIAS BIBLIOGRÁFICAS}

Carson, T. J.; Murray, G. F.; Wilcox, B. R. \& Starek, P. J. K. The role of surgery in tuberculous pericarditis. Ann. Thorac. Surg., 17(2):163-7, 1974.

Cegielski, J. P.; Devlin, B. H.; Morris, A. J.; Kitinya, J. N.; Pulipaka, U. P.; Lema, L. E.; Lwakatare, J. \& Reller, L. B. Comparison of PCR, culture and histopathology for diagnosis of tuberculous pericarditis. J. Clin. Microbiol., 35(12):3254-7, 1995.

Dooley, D. P.; Carpenter, J. L. \& Rademacher, S. Adjunctive corticosteroid therapy for tuberculosis: a critical reappraisal of the literature. Clin. Infect. Dis., 25(4):872-87, 1997.

Fowler, N. O. Tuberculous pericarditis. JAMA, 266:99-103, 1991.

Holman, E. \& Willet, F. Treatment of active tuberculous pericarditis by pericardiectomy. JAMA, 146:1-7, 1951.

Lasso, M. \& Pérez, J. Pericarditis due to multidrug resistant Mycobacterium tuberculosis in an HIV infected patient: case 
report and review of literature. Rev. Chil. Infect., 26(2):156$61,2009$.

Mayosi, B. M.; Ntsekhe, M.; Volmink, J. A. \& Commerford, P. J. Interventions for treating tuberculous pericarditis. Cochrane Database Syst. Rev., 4:CD000526, 2002.

Mayosi, B. M.; Volmink, J. A. \& Commerford, P. J. Pericardial disease: an evidence-based approach to diagnosis and treatment. In: Yusuf, S. Cairns, J. A.; Camm, A. J.; Fallen, B. J. (Eds.). Evidence-Based Cardiology. 2nd ed. London, BMJ Books, 2003. pp.735-48.

Mayosi, B. M.; Burgess, L. J. \& Doubell, A. F. Tuberculous pericarditis. Circulation, 112(23):3608-16, 2005.

Reuters, H.; Burgess, L. J. \& Doubell, A. F. Epidemiology of pericardial effusions at a large academic hospital in South Africa. Epidemiol. Infect., 133(3):393-9, 2005.

Reuter, H.; Burgess, L. J.; Schneider, J.; Van Vuuren, W. \& Doubell, A. F. The role of histopathology in establishing the diagnosis of tuberculous pericardial effusions in the presence of HIV. Histopathology, 48(3):295-302, 2006.

Sonneberg, F. A. \& Parker, S. G. Elective pericardiectomy for tuberculous pericarditis. Med. Decis. Making, 6(2):110-23, 1986.

Strang, J. I.; Kakaza, H. H.; Gibson, D. G.; Girling, D. J.; Nunn, A. J. \& Fox, W. Controlled trial of prednisolone as adjuvant in treatment of tuberculous constrictive pericarditis in Transkei. Lancet, 2(8573):1418-22, 1987.

Strang, J. I.; Kakaza, H. H.; Gibson, D. G.; Allen, B. W.; Mitchison, D. A.; Evans, D. J.; Girling, D. J.; Nunn, A. J. \& Fox, W. Controlled clinical trial of complete open surgical drainage and of prednisolone in treatment of tuberculous pericardial effusion in Transkei. Lancet, 2(8614):759-64, 1988.

Trautner, B. W. \& Darouiche, R. O. Tuberculous pericarditis: optimal diagnosis and management. Clin. Infect. Dis., 33(7):954-61, 2001.

Tuon, F. F.; Litvoc, M. N. \& Lopes, M. I. Adenosine deaminase and tuberculous pericarditis-- a systematic review with metaanalysis. Acta Trop., 99(1):67-74, 2006.

Yang, C. C.; Lee, M. H.; Liu, J. W. \& Leu, H. S. Diagnosis of tuberculous pericarditis and treatment without corticosteroid in a tertiary teaching hospital in Taiwan: a -14 years experience. J. Microbiol. Immunol. Infect., 38(1):47-52, 2005.

\author{
Dirección para correspondencia: \\ Dr. Oscar Tapia E. \\ Departamento de Anatomía Patológica \\ Facultad de Medicina \\ Universidad de La Frontera \\ Manuel Montt 112. \\ Código Postal 478-1176, Temuco \\ CHILE
}

Fono: 045-296530.

Email: otescalona@gmail.com

Recibido : 18-01-2012

Aceptado: 22-03-2012 